\title{
User-assisted Sparse Stereo-video Segmentation
}

\author{
Félix Raimbault, François Pitié and Anil Kokaram \\ Sigmedia Group \\ Dept. of Electronic and Electrical Engineering \\ Trinity College Dublin, Ireland \\ \{raimbauf, fpitie, anil.kokaram\}@tcd.ie
}

\begin{abstract}
Motion-based video segmentation has been studied for many years and remains challenging. Ill-posed problems must be solved when seeking for a fully automated solution, so it is increasingly popular to maintain users in the processing loop by letting them set parameters or draw mattes to guide the segmentation process. When processing multiple-view videos, however, the amount of user interaction should not be proportional to the number of views. In this paper we present a novel sparse segmentation algorithm for twoview stereoscopic videos that maintains temporal coherence and view consistency throughout. We track feature points on both views with a generic tracker and analyse the pairwise affinity of both temporally overlapping and disjoint tracks, whereas existing similar techniques only exploit the information available when tracks overlap. The use of stereo-disparity also allows our technique to process jointly feature tracks on both views, exhibiting a good view consistency in the segmentation output. To make up for the lack of high level understanding inherent to segmentation techniques, we allow the user to refine the output with a split-and-merge approach so as to obtain a desired view-consistent segmentation output over many frames in a few clicks. We present several real video examples to illustrate the versatility of our technique.
\end{abstract}

\section{Categories and Subject Descriptors}

I.4.6 [Image Processing and Computer Vision]: Segmentation

\section{General Terms}

sparse, motion segmentation, stereoscopic video, user interaction

\section{Keywords}

sparse, motion segmentation, stereoscopic video, user interaction

\section{INTRODUCTION}

The recent revival of the stereo movie industry, as well as the slow infiltration of stereoscopic content in consumer market call for new tools to automatise post-production tasks such as matte propagation for compositing of stereo videos. A key technology to enable the development of such tools is video segmentation, which has recently found many applications such as matting for cinema post-production [4], unsupervised learning [5], navigation for autonomous systems [1] and driver assistance systems [14].

Motion-based video segmentation aims at grouping together pixels belonging to objects with a similar motion in a video sequence, following Gesalt psychology principles that try to explain how humans perceive a coherent organisation of objects in a noisy world [20]. Defining what is an object is not trivial and each object could be represented by one or several clusters of pixels. For instance an articulated object (e.g. an actor) could be segmented into several parts exhibiting different motions (e.g. head, arms, torso) as illustrated in figure 1. This problem is known as setting the level of segmentation. Fully automated segmentation techniques [2, 27] need to infer the number of objects in the scene. However this is an ill-posed problem as the desired segmentation level ultimately depends on the application. Therefore, instead of segmenting a scene at a fixed level, a versatile technique would ideally output a hierarchy of clusters so that the level of fragmentation can be increased or decreased by users, depending on their needs.

It is important that each identified object consistently belongs to the same cluster along the whole video, i.e. that the segmentation is temporally coherent. Otherwise, manual corrections of the video segments would be required in a later stage. But it is still challenging for many state-of-the-art techniques to maintain a coherent grouping of pixels over many frames in real videos. In such sequences, lighting changes, object occlusions, complex camera motions and sensor noise are very common. These phenomena are challenging problems for motion estimation and feature point tracking $[24,25]$ which are at the core of most video segmentation algorithms. However, the most promising techniques enforce long-term temporal coherence by means of feature point trajectories or tracks to obtain a sparse representation of objects in the scene which span many frames $[4,5]$. After generating point trajectories, the first step towards segmentation is to gather similar tracks together into clusters as illustrated in figure 1.

For applications to stereo vision $[1,14]$ or stereo-3D cinema postproduction, coherence of the clustering across views should be enforced as well. Indeed, a cluster should correspond to a physical object that is imaged on both views. Left and right streams of a stereo sequence are very similar, so it would be more efficient to enable the user to process them jointly and yield a coherent grouping across views as shown in figure 1 . The main differences be- 
tween views arise in binocular half-occlusion areas which are hidden in only one view and should be segmented coherently with respect to the rest of the pixels. Besides, depth information that can be extracted from stereo pairs offers additional cues which can be combined with position, motion and colour to improve the quality of segmentation techniques [7, 14]. A general issue with segmentation is that it is an under-constrained problem, but the addition of depth or disparity-based constraints opens new possibilities that have not been explored that much in the literature so far.

The final goal of stereo-video segmentation is to assign every pixel to a cluster, i.e. generate a dense segmentation of every frame. However, temporal coherence and view consistency constraints must be enforced. Obtaining a sparse representation of a monoscopic video with feature trajectories has been employed as a first step to dense segmentation $[4,15,17]$. This allows exploitation of rich, long-term object position constraints and motion models that can be obtained from sparse video segments to yield a dense segmentation in a two-step sparse-to-dense approach. This approach seems very promising to enforce temporal coherence and overcome limitations due to analysis at local level in state-of-the-art dense video segmentation techniques [1]. Moreover, it should benefit from the addition of stereo cues to enable joint processing of a stereo sequence. Along these lines, we present in this paper a sparse stereovideo segmentation technique that can be employed as a stepping stone for dense stereo-video segmentation.

Our contributions: We propose a novel sparse stereo-video segmentation technique that builds up on the framework of Brox and Malik [5]. It extends it with a novel affinity measure on temporally disjoint tracks whereas state-of-the-art methods [12, 15] can only compare temporally overlapping tracks. We show that our technique increases temporal coherence of the output, enabling connection of objects even after full occlusion if their motion remains similar. Secondarily a consistent segmentation across views is yielded by processing jointly tracks generated on both left and right views in a single framework, making use of disparity-based constraints. To the best of our knowledge, this aspect has not been investigated in the sparse video segmentation literature heretofore. Finally we propose a user-assisted split-and-merge method allowing users to explore a hierarchy of clusters in a few clicks to generate an output with the level of segmentation that suits their needs without further processing. A binary tree of clusters in which each node represents a split step is obtained automatically beforehand by using a combination of algorithms employed in state-of-the-art techniques.

Organisation of the paper: Section 2 reviews previous similar approaches to sparse video segmentation. Section 3 details our technique for sparse segmentation of stereoscopic videos. The novel affinity allowing to compare both overlapping and disjoint tracks in stereo videos is presented in Section 3.2. The automatic hierarchical clustering method is described in section 3.3 before we present the user-assisted split-and-merge method in section 3.4. Experimental results are shown in section 4 before we conclude the paper.

\section{RELATED WORK}

Exploiting long-term point trajectories seems to be the most promising way of obtaining long-term temporally consistent clusters. Indeed, faster (or even real-time) techniques [1], based on frameto-frame propagation/relaxation using Optical Flow have troubles dealing with partial occlusions, spatially disconnected objects and large displacements. On the other hand, bottom-up segmentation
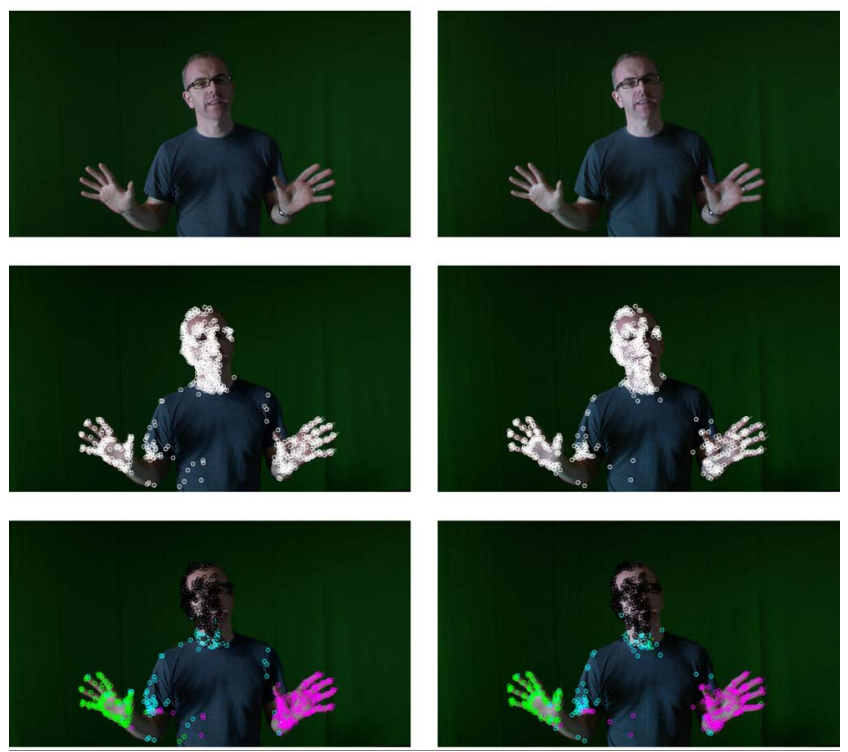

Figure 1: Sparse stereo segmentation on a stereo pair of frames from the green_screen sequence. Feature points on the actor can form a single cluster (middle row) or several clusters corresponding to different motions (bottom row).

techniques $[5,19,27]$, which are based on the analysis of feature point tracks, need to employ a tracker (such as KLT [24], LDOF [25], PV [22], or VT [3]) to generate trajectories as a preprocessing step. Not all feature point trackers perform equally in terms of spatial density and track length, but they all allow longer-term accurate correspondences between points than Optical Flow field.

Within a subclass of these track-based techniques, namely manifold clustering, completeness of all [27] or some [19] of the tracks is a common assumption. But in practice feature point trackers yield trajectories of variable lengths, that can be corrupted by noise; key points disappear and reappear due to occlusions, new objects entering the scene as well as illumination and viewpoint changes. To mitigate these problems, some techniques $[5,12,15]$ propose to analyse tracks by computing their pairwise affinity (i.e. measuring how similar tracks are to each other) during their time of overlap thereby allowing tracks of varying sizes to be clustered efficiently. Once pairwise track affinity computed, many techniques [5, 27] employ spectral clustering algorithms [11, 23] that analyse the eigenvalues of the affinity matrix to form groups of similar tracks. Other energy minimisation techniques based on track affinities can be used [15]. These methods can deal with partial occlusions and tracking loss but break down fully occluded objects. A track repair mechanism [21] would be needed to connect temporally disjoint but similar tracks. Bridging the gap between track repair and clustering, we propose to compute affinities for both disjoint and overlapping tracks to mitigate problems with fully occluded objects in state-of-the-art sparse video segmentation techniques.

Affinity is usually defined from computation of a pairwise track distance. A key contribution to the field [5] has been to note that the actual information obtained from track comparison does not reside in the common motion but in the motion difference (e.g. it is not possible to differentiate a person sitting from the background until they stand up). Consequently distances between two tracks are defined as the maximum difference of their motion over time. Sim- 

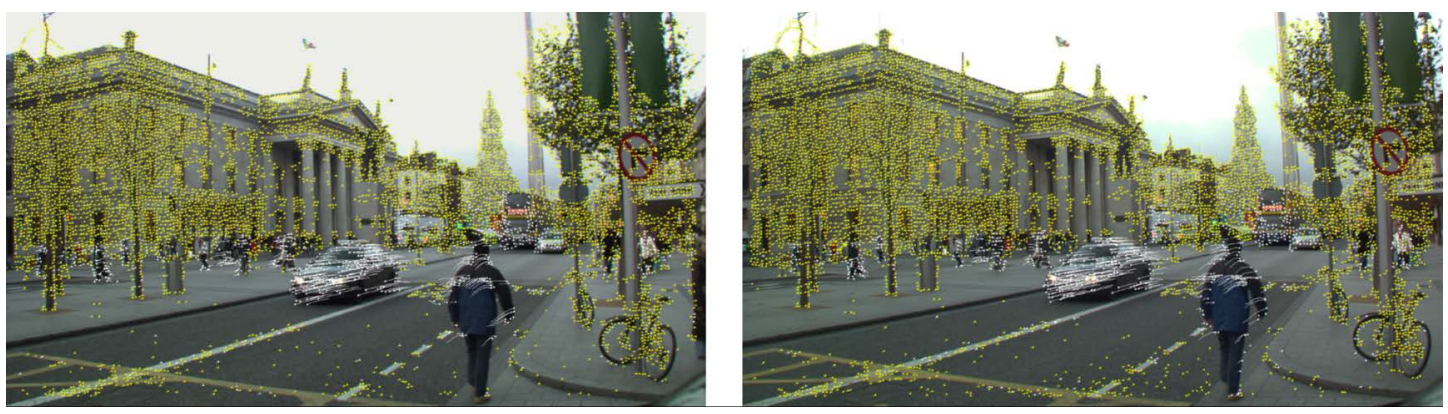

Figure 2: Bi-layer separation on a stereo pair from the gpo_fixed video. Points in yellow are ignored in subsequent segmentation.

ilarly, Fragkiadaki et al. [12] add a term based on the maximum stereo-disparity difference between tracks in one view of a stereo video alongside the maximum velocity difference. Occlusion reasoning in the form of depth ordering constraints has been added to the pairwise affinity framework [15] to further constrain the problem. However depth ordering is assumed constant over the video. We exploit stereo disparity as a more flexible natural depth ordering constraint for stereo videos $[9,12]$. Our technique is also able to compare two trajectories that do not lie in the same view by adding corresponding disparity offsets to tracked feature point positions.

A common problem in most techniques is the lack of high level understanding of a video scene. Two-granularity tracking [12] combines the bottom-up approach of Brox and Malik [5] with a topdown model-driven technique to track pedestrians in crowded environment. The finer granularity of point tracks allows refinement of the model-based technique in case partial occlusions occur. Balancing cues such as appearance and geometry [2] or colour and depth [7] can prove very hard and need manual tuning. In Baugh and Kokaram [4] the user is asked to draw a matte every ten frames to provide higher level information on the colour and motion of objects of interest. Parameter tuning can be time consuming and frustrating for the user, so we have tried to make most parameters adaptive. However we ask users to choose the desired level of segmentation that fits their needs which only requires a few clicks.

Determining the number of clusters in the sequence is a difficult problem that requires high level understanding of the scene as well. Many automatic solutions have been proposed, exploiting spatial regularity [5], the Akaike Infomation Criterion [2], distribution of the model hyperparameters [13], spectral graph theory [27], etc. But none can predict what is the actual number of clusters desired by the user, which is application-dependant (e.g. only two clusters are necessary for foreground/background separation [14]) so it may be more practical to let the user set it as a parameter [15]. In some applications, however, over-segmentation is acceptable as long as it provides long-term associations between points while respecting object boundaries, so that the number of clusters need not to match exactly the number of objects in the scene. Our approach to that problem is to generate a hierarchy of clusters that can be navigated by the user to obtain the required segmentation result.

Reviewing in details how sparse-to-dense approaches [4, 15, 17] turn clusters of tracks into dense video segments is out of the scope of this paper as we present a sparse segmentation technique. Interested readers can refer to the provided references for further information. Now we have reviewed the current state of the art of sparse video segmentation, the next section delves into details of our ap- proach to solve the sparse stereo-video segmentation problem.

\section{PROPOSED SOLUTION}

This section details the steps of our sparse stereo-video segmentation technique. Firstly, section 3.1 explains the preprocessing stages necessary to obtain a $6 \mathrm{D}$ feature vector at each tracked points in the whole stereo sequence and how static background points are discarded from further processing. These feature point tracks are the input of the sparse segmentation algorithm per se. Section 3.2 shows how pairwise affinity scores are computed between pairs of both overlapping and disjoint tracks in left and right views. Then section 3.3 describes the divisive-agglomerative algorithm to generate a hierarchy of clusters via over-segmentation using spectral clustering before an agglomerative step merges pairs of clusters one by one. Finally section 3.4 explains how it is then expanded and refined during a user-assisted split-and-merge step which allows users to select the desired level of segmentation of the output.

\subsection{Preprocessing}

Our method uses a sparse representation of a stereo video obtained via feature point tracking and disparity estimation. The idea is to store $2 D$ position and stereo-disparity information, as well as their temporal derivatives for each feature point along their trajectory. Thus we obtain a $6 \mathrm{D}$ feature vector at each point by combining cues on position $X$ and velocity $V$ :

$$
F=[\underbrace{x, y, d}_{X}, \underbrace{\frac{\partial x}{\partial t}, \frac{\partial y}{\partial t}, \frac{\partial d}{\partial t}}_{V}]
$$

Following Gesalt psychology principles we seek to cluster together proximate points exhibiting a similar motion, following the framework of previous techniques $[5,12]$ and exploit the feature vectors $F$ to compute pairwise affinities between tracks (see section 3.2).

We employ the KLT feature tracker [24] on both views independently and off-the-shelf state-of-the-art disparity estimator available in Ocula ${ }^{1}$, a stereo software suite for a compositing software for movie post-production, Nuke ${ }^{2}$. We also estimate local Optical Flow fields with Nuke to discard the most noisy KLT tracks that exhibit a drastically different displacement from corresponding local motion vectors. A stereo feature tracker [16] that simultaneously tracks points in both cameras could also be used advantageously in our technique, except that no points would be tracked on binocular half occlusion areas (i.e. points visible in one view only).

\footnotetext{
${ }^{1} \mathrm{http}: / /$ www.thefoundry.co.uk/products/ocula/

${ }^{2}$ http://www.thefoundry.co.uk/products/nuke/
} 
Information about 2D positions of tracked points is extended with their stereo-disparity information to form a $3 \mathrm{D}$ feature vector noted $X$. We thus obtain a 3D representation for each point similar to Duan et al. [9]. We use it to favour grouping together points within a depth layer in our clustering algorithm (see section 3.3), as disparity is inversely proportional to the depth of the underlying 3D point. The position of a feature point can be inaccurate and wander across depth layers, causing spurious fluctuations of the disparity value. So we apply a temporal median smoothing filter on the disparity values along trajectories, using the previous and next frames.

Temporal derivatives of the position (i.e. velocity) and of the disparity (that we call depth variation) are then computed and denoted as a 3D feature vector $V$. The 2D velocities are further processed to remove the influence of the camera motion noise. This step is similar to the use of video stabilisation in the track repair technique [21]. Indeed velocities of temporally disjoint tracks must be comparable in the measure we detail in section 3.2, which would not be the case if they were corrupted by motion jitter (e.g. when the camera is handheld). Our procedure estimates a Dominant Motion Vector [18] at each frame, on velocities of feature points only. It is then subtracted from all velocity values, on each view separately. Finally the corrected velocities and depth variation values are temporally smoothed out with a two-pass (forward and backward) onetap IIR filter with parameter $\alpha_{M}=0.75$ to increase robustness in subsequent computation involving $V$.

Once velocities registered and smoothed, basic bi-layer segmentation is used to label points that are moving due to camera motion as background (see figure 2). These points have a computed velocity of 0 as velocities are registered to the camera motion. So we can detect them by a simple thresholding of the quantity of motion along time. If the maximum of their squared motion amplitude remains below $\theta_{M}=2$ along the trajectory, we classify the track as belonging to the background. Static background tracks are discarded in further processing as we are mainly interested in segmenting dynamic objects. However we do not discard any tracks if the amount of foreground trajectories is less than $P_{M}=50 \%$ of the total number of tracks. This avoids inaccurate background labelling, which can happen under camera zooming, as our motion registration step is translational only. The bi-layer pre-segmentation step is useful to reduce computation speed and balance the size of clusters (the presence of a very large cluster can bias some clustering techniques).

In section 3.2 pairwise affinities are computed between each and every pair of tracks, regardless of the view in which they reside. To enable that computation, a key point of our technique is computing of a view counterpart position vector for each point:

$$
X^{\prime}=(x+d, y,-d)
$$

The counterpart velocity $V^{\prime}$ stems from $X^{\prime}$ as explained previously. Given $F$ and $F^{\prime}$ at each tracked point we can explain in details how affinity values between non-static tracks are computed and gathered in an affinity matrix.

\subsection{Computation of the Affinity Matrix}

Along each trajectories we compute feature vectors $F=(X, V)$ and $F^{\prime}=\left(X^{\prime}, V^{\prime}\right)$ as explained in section 3.1. From now on, for notational simplicity we note all feature vectors without prime regardless, but assume that when given a pair of tracks $(i, j)$ residing in different views, one of the tracks (e.g. the first one, i) uses its view counterpart feature vector $F_{i}^{\prime}$. For every track $i$, the corresponding feature at frame $t$ is then noted $F_{i}^{t}=\left(X_{i}^{t}, V_{i}^{t}\right)$.

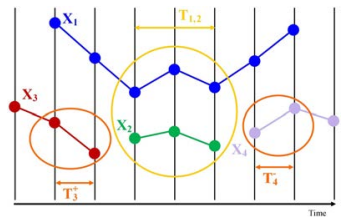

Figure 3: Affinity is computed on both overlapping (e.g. 1 and 2) and disjoint tracks (e.g. 3 and 4).

As stated in section 2, previous methods compute the pairwise affinity between overlapping tracks $(i, j)$, using feature vectors taken at frames $t \in T_{i, j}$ for which both tracks exist (e.g. tracks 1 and 2 in figure 3 are overlapping during 3 frames). A key novelty of our method is to compute affinities between disjoint tracks $(i, j)$ as well, using feature vectors taken at the extremity of each track. If track $i$ stops before track $j$ begins, we use features at frames in sets $T_{i}^{+}$at the end of track $i$ and in $T_{j}^{-}$at the beginning of track $j$. Conversely $T_{i}^{-}$and $T_{j}^{+}$are employed if track $j$ ends before track $i$ begins (e.g. tracks 3 and 4 in figure 3 are disjoint). If $T_{i, j}$ covers less than two frames, we consider the tracks as being disjoint. We use $\Delta_{T}=3$ frames at the extremity of disjoint tracks. Whenever a track is shorter than $\Delta_{T}$, only the smallest amount of available frames is considered in a pairwise computation involving this track.

\subsubsection{Pairwise Distance Matrix}

Following the framework presented in previous works [5, 15, 27] we perform track clustering on a pairwise affinity matrix indicating how similar tracks are to each other. The affinity matrix is derived from a pairwise normalised distance matrix $D$ made of two terms:

$$
D(i, j)=\left(1+\frac{D_{s}(i, j)-m_{s}}{\sigma_{s}}\right)\left(\frac{D_{t}(i, j)-m_{t}}{\sigma_{t}}\right)
$$

For all pairs of tracks $(i, j)$. The matrix $D_{s}$ is the spatial pairwise distance and $D_{t}$ is the temporal pairwise distance. The normalising term $\sigma_{u}$ for $u \in\{s, t\}$ is computed as the standard deviation of all elements in the corresponding matrix $D_{u}$ excluding the diagonal. Similarly $m_{u}$ is the minimal value in $D_{u}$. We found that automated normalising step to work well for all the sequences we have tested. Pairwise track distances can only compare the compatibility of trajectories on the basis of translational motion models [5], valid only for points that are close enough in space. To reduce the effects of that approximation we normalise the distance using $D_{s}$ so that only proximate points can generate high affinities. One is added to the leftmost term so that when the normalised spatial distance is null, a low distance is obtained only if the temporal distance is low.

\subsubsection{Spatial distance}

The pairwise spatial normalisation term $D_{s}(i, j)$ in equation (3) is computed as follows:

$$
D_{s}(i, j)= \begin{cases}\mu_{T_{i, j}}\left(\left\|X_{i}-X_{j}\right\|\right), & \text { if }\left|T_{i, j}\right| \geq 2 \\ \left\|\mu_{T_{i}^{a}}\left(X_{i}\right)-\mu_{T_{j}^{b}}\left(X_{j}\right)\right\|, & \text { otherwise. }\end{cases}
$$

We define the mean function as $\mu_{T}(X)=\frac{1}{T \mid} \sum_{t \in T} X^{t}$ and $|T|$ as the number of elements in the set $T$. For disjoint tracks, $a$ and $b$ correspond to the appropriate sign + or - depending on which track precedes the other. This term ensures that only overlapping tracks which remain close to each other on average along their common lifespan or disjoint tracks which reside nearby at their extremities can yield a high affinity. Note that points that are far apart can still be grouped in the same cluster via transitivity of the affinity graph. 


\subsubsection{Temporal distance}

The pairwise temporal distance term $D_{t}(i, j)$ in equation (3) is computed as follows:

$$
D_{t}(i, j)= \begin{cases}\max _{t \in T_{i, j}}\left(\left\|V_{i}^{t}-V_{j}^{t}\right\|\right), & \text { if }\left|T_{i, j}\right| \geq 2 \\ \left\|\mu_{T_{i}^{a}}^{a}\left(V_{i}\right)-\mu_{T_{j}^{b}}^{a}\left(V_{j}\right)\right\|, & \text { otherwise. }\end{cases}
$$

Where $a$ and $b$ are defined as in equation (4). The average function $\mu^{-}$excludes one frame at the beginning of a track as the velocity is not defined on the first frame, but considers the $\Delta_{T}$ following frames. And $\mu^{+}$is the same as $\mu$. The distance enforces the formulation of Brox and Malik [5] for overlapping tracks, with the addition of the depth variation term. Moreover, high affinity is assigned to disjoint tracks which exhibit a similar motion on average at their extremities. Note that only stationary disjoint tracks would legitimately have a high affinity in our formulation. It is important to repeat that the values computed for disjoint tracks assume that the effects of camera jitter are removed in the velocity terms (see section 3.1), following the track repair framework [21].

\subsubsection{Temporal decay}

The approximation of stationarity made in equation (5) for disjoint tracks holds only for tracks that are not too far apart in time, so we define a decay factor $\lambda$ to lower the affinity of disjoint tracks as the length of the temporal gap $g_{i, j}$ between them widens:

$$
\lambda(i, j)= \begin{cases}1, & \text { if }\left|T_{i, j}\right| \geq 2 \\ \exp \left(-\frac{g_{i, j}^{2}}{2 \sigma_{t}^{2}}\right), & \text { otherwise. }\end{cases}
$$

Where $\sigma_{t}$ equals the number of frames in the sequence, as we have obtained better results when not constraining the value too much.

\subsubsection{Pairwise Affinity Matrix}

The affinity matrix $A$ is computed as a mixture of a distance-based affinity matrix $A_{D}$ computed from $D$, and a neutral value $\mu_{D}$ to which disjoint tracks that are far apart converge:

$$
A(i, j)=\lambda(i, j) A_{D}(i, j)+(1-\lambda(i, j)) \mu_{D}
$$

Where $A_{D}(i, j)=\exp \left(-D(i, j)^{2}\right)$ with $D$ from equation (3). And $\mu_{D}$ is the mean value of the elements in $A_{D}$ corresponding to overlapping tracks only, excluding the diagonal. Then affinity values are normalised to $[0,1]$. The mixing weights $\lambda$ are the decay values for disjoint tracks from equation (6). This ensures that more credit is given to affinities of disjoint tracks that are not too far apart in time while mitigating more dubious values (viz. when tracks are far apart in time) with a neutral scalar computed from more reliable affinities of overlapping tracks. Note that the average of values obtained by comparing both left-to-right and right-to-left view counterpart tracks is assigned to the final values in $D_{s}$ and $D_{t}$ constituting the distance matrix $D$.

We have now explained in detail how to compute affinity values between all pairs of tracks. In section 3.3 we move on to the segmentation algorithm which uses that information to form groups of clusters and show that the output respects both temporal coherence and view consistency constraints mentioned in section 1 .

\subsection{Divisive-Agglomerative Clustering}

This section describes how we construct a hierarchy of track clusters via a divisive-agglomerative clustering approach [2]. The divisive step uses spectral clustering with Normalised Cuts [23] on

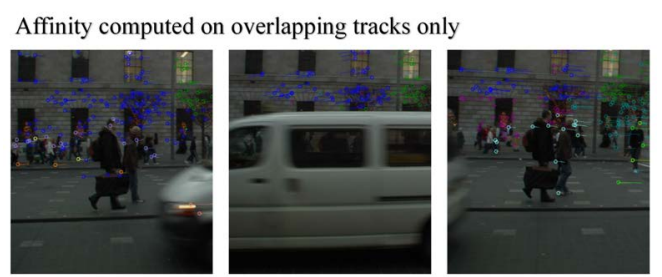

Affinity computed on both disjoint and overlapping tracks

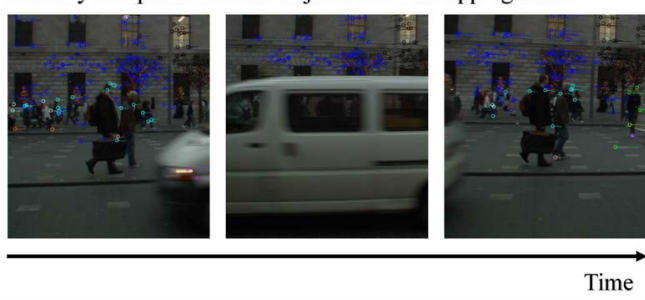

Figure 4: Output of divisive step of section 3.3.1 on the gpo_track_2 sequence (details). Using affinity on both overlapping and disjoint tracks allows recovery from full occlusion.

the pairwise affinity matrix computed as explained in section 3.2 to over-segment the stero video and produce a large number of small but consistent clusters and the following agglomerative step uses Min-max Cut linkage [8] to merge all clusters one by one, thereby forming a binary tree where each node corresponds to a split move when reading the tree from the root.

\subsubsection{Divisive Step}

Once the pairwise affinity matrix $A$ computed for all pairs of tracks, we analyse its eigenvalues to find clusters of tracks. Dal Mutto et al. [7] compare several clustering algorithms to segment stereo images using geometry and colour. They found that the most reliable and efficient technique on their data is spectral clustering with the Nyström method [11]. It is an approximation to solve the eigenfunction problem of Normalised Cuts [23] more efficiently, making it scalable to process big datasets. In our experiments we used the recursive two-way Ncut algorithm [23] with a modified stopping criterion for a better control on the size and coherence of the clusters. This divisive step yields an over-segmentation of the tracks, generating small but very consistent clusters.

The two-way Ncut algorithm [23] boils down to thresholding the eigenvalue corresponding to the Fielder vector associated with the second smallest eigenvalue of the normalised Laplacian matrix derived from the affinity matrix $A$. Thereby it finds a splitting point that optimally partitions the underlying graph in two. Each subgraph is then sub-partitioned iteratively until a stopping criterion is reached. It is hard to attach a physical meaning to the original criterion [23]. So we adopted a more pragmatic approach that directly controls the coherence of the clusters in terms of affinity values. First we define the binary matrix $B$ which locates pairs of tracks with low affinities that should not be grouped together:

$$
B(i, j)= \begin{cases}1, & \text { if } A(i, j) \geq \theta_{A} \\ 0, & \text { otherwise }\end{cases}
$$

Where the threshold $\theta_{A}$ gives an indication on what is a high affinity value. We define $\theta_{A}$ as the mean value of values of $A$ greater that the mean value of $\mathrm{A}$ excluding the diagonal. This adaptive threshold yields satisfying results for all the sequences we have tested. At the $k^{\text {th }}$ iteration of $t w o-w a y ~ N c u t$, if the number of feature tracks 


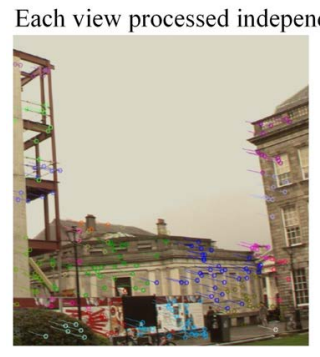

Both views processed jointly

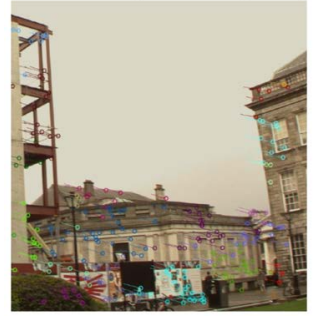

Left view
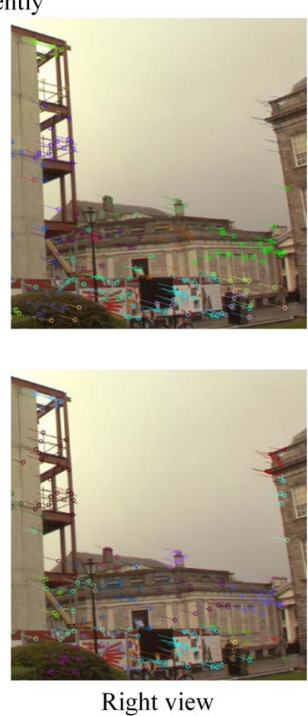

Figure 5: Output of divisive step of section 3.3.1 on the building_site sequence (details). The shape of the clusters differ significantly on the top row (in the right area especially) and are much more consistent on the bottom row.

$n_{k}$ in the current cluster under scrutiny is greater than $n_{\min }=3$, we pursue the partitioning of the weighted sub-graph only if the amount of confident pairs of tracks $\beta_{k}=\frac{1}{n_{k}\left(n_{k}-1\right)} \sum_{i \neq j} B_{k}(i, j)$ is greater than a threshold $\theta_{H}=0.2$, where $B_{k}$ stores the values of $B$ for the sub-graph. Finally small clusters which sizes are lesser or equal to $n_{\min }$ are put together and the algorithm is run on that sub-graph once more, to try and merge them back together.

We have compared the output of the divisive algorithm when using affinities on overlapping tracks only and all tracks and found out that temporal coherence is improved with the latter approach. As can be seen on figure 4 recovery from full occlusion is only possible if disjoint tracks can yield a high affinity value as well. We also tried to run the divisive clustering on each view of a stereo video separately and compared it with our joint approach. As illustrated in figure 5 the shape of clusters can differ significantly from one view to the other when processed separately whereas a much greater view consistency is attained by our approach.

\subsubsection{Agglomerative Step}

At the end of the divisive step we separate clusters that are disjoint in time. The following agglomerative step uses the Min-max Cut linkage technique $[2,8]$ to iteratively merge all clusters together. At each iteration the algorithm merges the clusters $C_{p}$ and $C_{q}$ that yield the greatest linkage score:

$$
L(p, q)=\frac{S(p, q)}{S(p, p) S(q, q)}
$$

Where $S(p, q)=\sum_{i \in C_{p}, j \in C_{q}} A(i, j)$ denotes the similarity between clusters. The output of our clustering method is then a hierarchy of consistent clusters that can be represented as a binary tree in which each node corresponds to a split step, when reading from the root.

It is difficult to set an appropriate fixed level of segmentation corresponding to a defined amount of split steps when traversing the tree. Therefore we ask the user to explore possible split moves

before merging back some clusters. This user-assisted split-andmerge step is explained in section 3.4 before application of the technique is shown on examples in section 4.

\subsection{User-assisted Split-and-Merge}

Up to now we have not defined the number of clusters in the final output of our technique. As stated in section 2 defining automatically the appropriate number of clusters is a challenging problem for which a variety of ad-hoc solutions have been proposed. In fine the desired number of clusters is application-dependant (e.g. foreground/background segmentation requires two clusters, matte propagation can only require one or several specific object(s) to be segmented out of the rest of the sequence) so we have decided to let the user choose a suitable level of segmentation.

Determining a fixed level at which the tree defined in section 3.3 is pruned as in Angeli and Davison [2] can generate a solution that is not optimal for the purpose of the user, i.e. over or undersegmented. Remember that the track comparison method we employ is only able to model translations. The linkage measure we use for grouping in section 3.3.2 can then generate spurious associations, for instance under heavy camera rotation around the optical axis where the local motion of tracks at the centre of the scene differs drastically from the motion of tracks at the edges.

Therefore we propose to provide users with an interactive tool that allows them to explore the clustering hierarchy to correct under or over-segmentation errors and reach the output that suits their needs. The technique can be implemented as a graphical user interface enabling users to play back the video while refining the segmentation. One click on a marker at the centroid of a cluster at a given frame would allow to split it in two, along all its duration and on both views until there is no under-segmented clusters remaining (see the output of the split step in the top row of figure 6), and a selection tool would then allow the user to click on several cluster to be merged together (see the output of the merge step on the bottom row of figure 6). If dense segmentation is desired, sparse segmentation can be used as a preview of the final output that can be generated in a fraction of the time required for a dense result.

In our tests we have implemented the technique as a command line tool, but in section 4 we refer to the number of clicks and selections made to segment a scene as an indicator of the amount of user interaction needed for a given segmentation task.

\section{EXPERIMENTS AND RESULTS}

Now we have explained how our technique works we analyse userassisted segmentation results on a few sequences extracted from a stereo-video database available online [6]. We keep 100 frames for every sequence in our tests. We also show the misclassification score of our automatic method applied to the Hopkins 155 dataset [26] compared to two state-of-the-art sparse segmentation techniques. For all our tests we have used the parameter values as given throughout section 3 . Videos corresponding to the results presented here as well as additional sequences can be viewed on our website at http://www. sigmedia.tv/Misc/SSVS2013.

We experimented on the Hopkins 155 dataset [26] to test the basic performance of our automatic divisive-agglomerative technique on overlapping tracks only in short monoscopic videos. Table 1 shows the corresponding misclassification scores of our technique when the final number of clusters is set to the ground truth, com- 


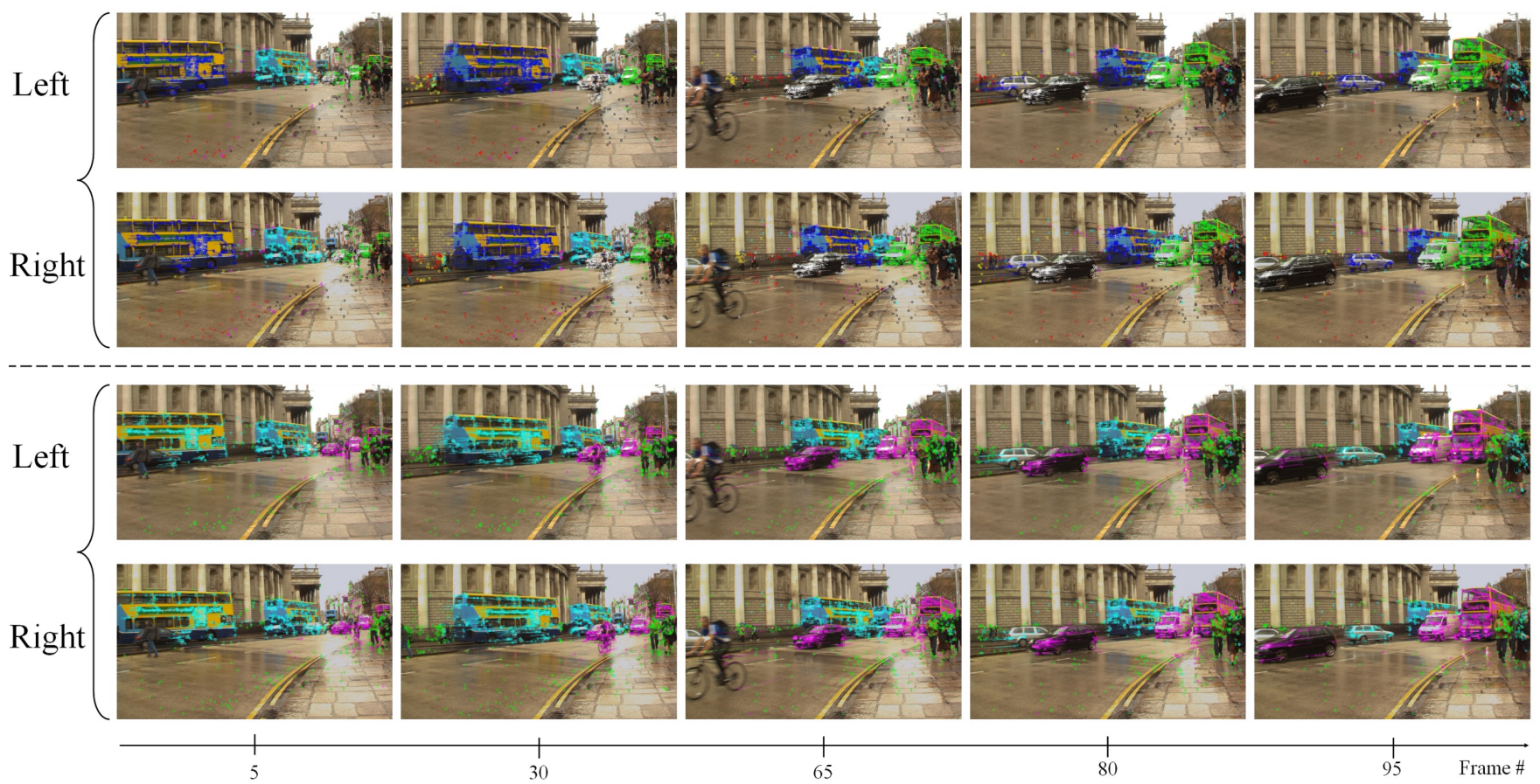

Figure 6: traffic sequence - user-assisted output after 7 split steps (top rows) and after 3 merge steps (8 selections) (bottom rows).

pared to two recent methods $[10,19]$. On the traffic and articulated sequences our results are in par with state-of-the-art scores but some sequences with heavy rotation of the camera among the chequerboard sequences obtain a very poor score, because the agglomerative step produces erroneous associations, as explained in section 3.4. There are many such sequences in that database, so the overall score suffers from that problem. It would be interesting to test our technique on the database presented in Brox and Malik [5] for tracks of variable lengths, which we plan to do in the future.

In figure 7 we show the output of automatic clustering presented in section 3.3, with the number of clusters set to 5. When computing affinities on overlapping tracks only, we set the affinity of non-overlapping tracks to 0 which indicates the lack of information regarding their association [12]. When computing the affinities on both overlapping and disjoint tracks with our novel affinity measure it can be seen that the segmentation is much more temporally coherent. We also show on that example points estimated as background during preprocessing in gray.

The next three figures compare the output of our automatic divisiveagglomerative algorithm with a set number of clusters to the output obtained by user-assisted exploration of the cluster hierarchy described in section 3.4. The examples are presented in increasing order of difficulty in terms of required user interaction. In the video presented in figure 8 motions of the tracks on the walking person and the rotating sphere are locally similar, causing wrong associations between the two objects, even though differences in position and disparity allows for separation of most of the tracks. Splitting the spurious clusters allows to efficiently differentiate between the two objects. In the sequence presented in figure 9, variations in the speed of the camera undergoing a panning movement causes the motion of points in the background to differ significantly over time and therefore to be over-segmented. User-assisted exploration allows to merge those points in one cluster and differentiate between the various moving objects in the scene. Finally the sequence shown in figure 10 contains two main challenges. Firstly it is difficult to separate the background from the head of the actor, as both are immobile for a long period of time, and many tracks are terminated when he starts moving his head. Secondly the area in between both actors is problematic as they are close to each other and keep the upper-part of their torso almost immobile as well. User-assisted exploration, necessitating to over-segment the area connecting both actors ultimately allows to generate a meaningful segmentation separating both actors and the background. In all sequences view consistency is enforced very well, so that in our tests the user never had to connect clusters residing in different views.

\section{CONCLUSIONS AND FUTURE WORK}

We present a novel user-assisted sparse stereo-video segmentation technique able to efficiently segment stereo sequences while maintaining good temporal coherence and accurate view consistency. Temporal coherence is enforced throughout the video with a novel affinity measure on disjoint tracks improving the state of the art by allowing recovery from total occlusions, whereas only partial occlusions could be dealt with in previous works. View consistency is maintained by joint processing of features in both views as well as using disparity as a cue when computing affinity. A novel userassisted split-and-merge refinement step gives our method great versatility as illustrated on various real sequences in section 4 . It would be interesting to exploit colour cues along tracks in addition to disparity and position, especially when considering disjoint tracks [21]. To overcome inherent model limitations of our technique and allow more accurate processing of sequences with camera rotation we plan to replace our agglomerative step by a merge step based on affine motion estimation $[4,5]$. Our current endeavours consist in extending the technique with a sparse-to-dense approach $[4,15,17]$ to generate semi-automatic dense segmentation of stereo videos while keeping only minimal user interaction. 

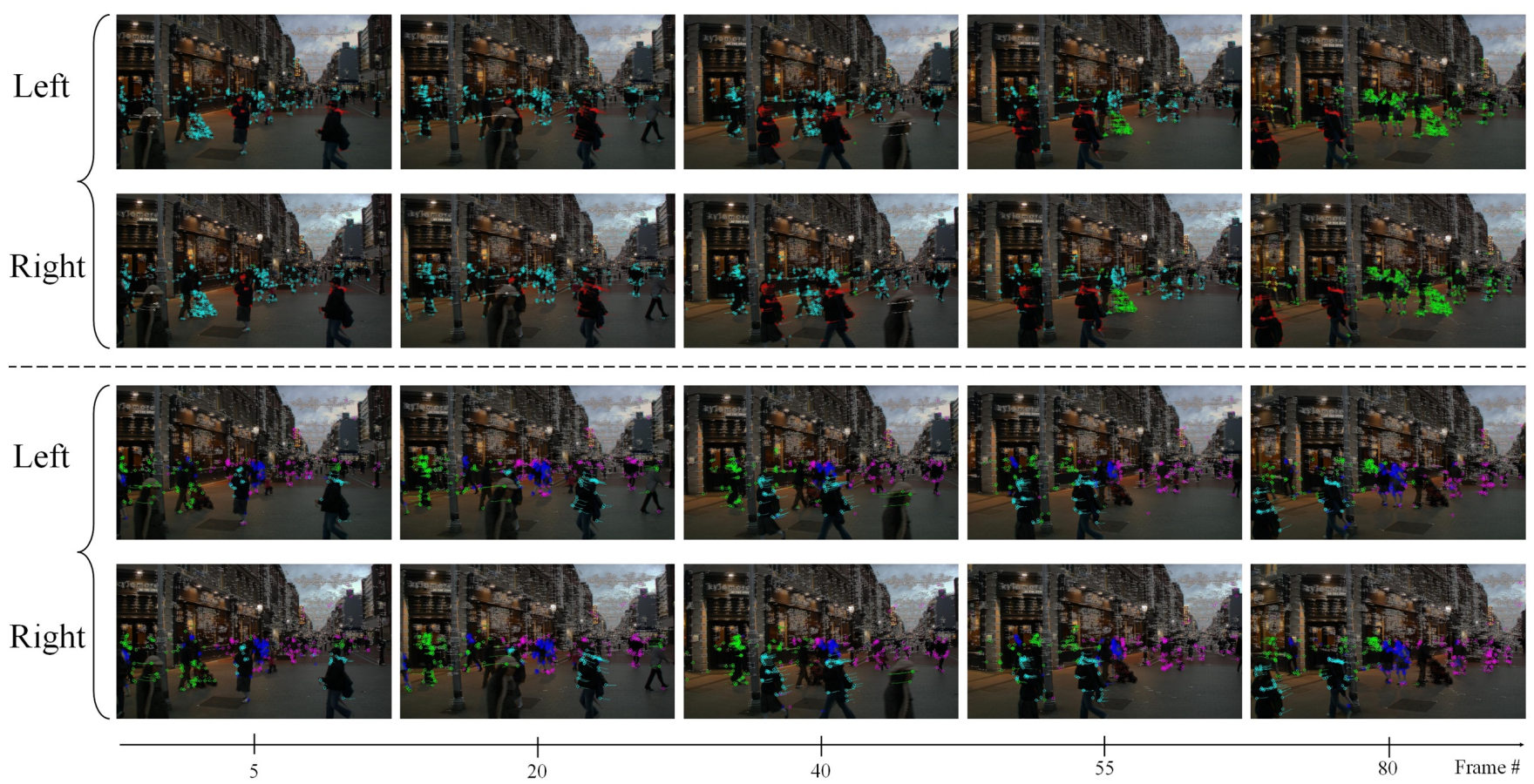

Figure 7: kylemore sequence - results of automatic divisive/agglomerative clustering with number of clusters set to 5 , with overlapping affinities only (top rows) and both overlapping and disjoint affinities (bottom rows).

\begin{tabular}{|c|c|c|c|c|c|c|c|}
\hline \multicolumn{2}{|c|}{} & \multicolumn{3}{|c|}{2 motions } & \multicolumn{3}{c|}{3 motions } \\
\hline sequences & method & $\mathrm{ALC}_{\mathrm{sp}}[19]$ & $\mathrm{SSC}[10]$ & our method & $\mathrm{ALC}_{\mathrm{sp}}[19]$ & $\mathrm{SSC}[10]$ & our method \\
\hline \multirow{2}{*}{ chequerboard } & mean & 1.49 & 1.12 & 12.47 & 5.00 & 2.97 & 14.46 \\
\cline { 2 - 8 } & median & 0.27 & 0.00 & 0.13 & 0.66 & 0.27 & 15.94 \\
\hline \multirow{2}{*}{ traffic } & mean & 1.75 & 0.02 & 0.29 & 8.86 & 0.58 & 1.48 \\
\cline { 2 - 8 } & median & 1.51 & 0.00 & 0.00 & 0.51 & 0.00 & 0.00 \\
\hline \multirow{2}{*}{ articulated } & mean & 10.7 & 0.62 & 6.74 & 21.08 & 1.42 & 3.55 \\
\cline { 2 - 7 } & median & 0.95 & 0.00 & 0.00 & 21.08 & 1.42 & 3.55 \\
\hline
\end{tabular}

Table 1: Results on the Hopkins 155 database in \% of misclassification.

\section{ACKNOWLEDGEMENTS}

We would like to thank the anonymous reviewers for their helpful comments. This work has been funded by Science Foundation of Ireland (SFI) as part of Project 08/IN.1/I2112, Content Aware Media Processing (CAMP).

\section{REFERENCES}

[1] A. Abramov, K. Pauwels, J. Papon, F. Worgotter, and B. Dellen. Real-time segmentation of stereo videos on a portable system with a mobile GPU. IEEE Transactions on Circuits and Systems for Video Technology, 22(9):1292-1305, 2012.

[2] A. Angeli and A. Davison. Live feature clustering in video using appearance and 3D geometry. In British Machine Vision Conference, pages 41.1-41.11, 2010.

[3] G. Baugh and A. Kokaram. A Viterbi tracker for local features. In SPIE Visual Communications and Image Processing, pages 7543-23, 2009.

[4] G. Baugh and A. Kokaram. Semi-automatic motion based segmentation using long term motion trajectories. In IEEE International Conference on Image Processing, pages 3009-3012, 2010.
[5] T. Brox and J. Malik. Object segmentation by long term analysis of point trajectories. In European Conference on Computer Vision, pages 282-295, 2010.

[6] D. Corrigan, F. Pitié, V. Morris, A. Rankin, M. Linnane, G. Kearney, M. Gorzel, M. O’Dea, C. Lee, and A. Kokaram. A video database for the development of stereo-3D post-production algorithms. In European Conference on Visual Media Production, pages 64-73, 2010.

[7] C. Dal Mutto, P. Zanuttigh, G. Cortelazzo, and S. Mattoccia. Scene segmentation assisted by stereo vision. In International Conference on 3D Imaging, Modeling, Processing, Visualization and Transmission, pages 57-64, 2011.

[8] C. Ding, X. He, H. Zha, M. Gu, and H. Simon. A min-max cut algorithm for graph partitioning and data clustering. In IEEE International Conference on Data Mining, pages 107-114, 2001.

[9] T. Duan, W. Huang, and M. Constable. Detecting the presence of stationary objects from sparse stereo disparity space. In Pacific Rim Symposium on Image and Video Technology, pages 15-20, 2010.

[10] E. Elhamifar and R. Vidal. Sparse subspace clustering. In 

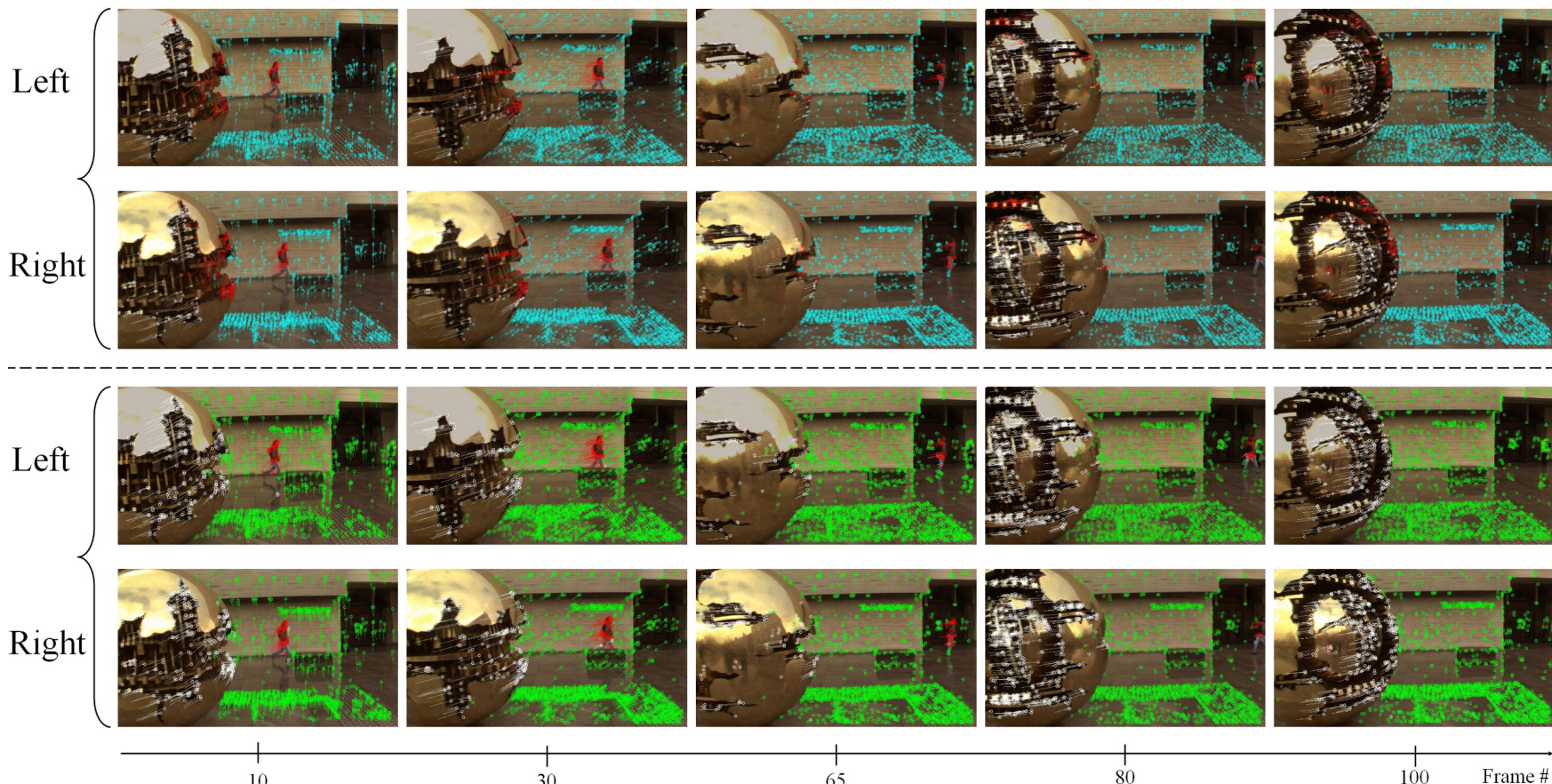

30

65

80

$100 \quad$ Frame \#

Figure 8: sphere_2 sequence - results of automatic divisive/agglomerative clustering with number of clusters set to 3 (top rows) and after user-assisted split-and-merge producing 3 clusters (4 split steps, 1 merge steps, 3 selections) (bottom rows).

IEEE Conference on Computer Vision and Pattern

Recognition, pages 2790-2797, 2009.

[11] C. Fowlkes, S. Belongie, F. Chung, and J. Malik. Spectral grouping using the Nyström method. IEEE Transactions on Pattern Analysis and Machine Intelligence, 26(2):214-225, 2004.

[12] K. Fragkiadaki, W. Zhang, G. Zhang, and J. Shi. Two-granularity tracking: Mediating trajectory and detection graphs for tracking under occlusions. In European Conference on Computer Vision, pages 552-565, 2012.

[13] V. Karavasilis, K. Blekas, and C. Nikou. Motion segmentation by model-based clustering of incomplete trajectories. In European Conference on Machine Learning and Knowledge Discovery in Databases, pages 146-161, 2011.

[14] J. Klappstein, T. Vaudrey, C. Rabe, A. Wedel, and R. Klette. Moving object segmentation using optical flow and depth information. In Pacific Rim Symposium on Advances in Image and Video Technology, pages 611-623, 2009.

[15] J. Lezama, K. Alahari, J. Sivic, and I. Laptev. Track to the future: Spatio-temporal video segmentation with long-range motion cues. In IEEE Conference on Computer Vision and Pattern Recognition, pages 3369-3376, 2011.

[16] K. Ni and F. Dellaert. Stereo tracking and three-point/one-point algorithms - A robust approach in visual odometry. In IEEE International Conference on Image Processing, pages 2777-2780, 2006.

[17] P. Ochs and T. Brox. Object segmentation in video: A hierarchical variational approach for turning point trajectories into dense regions. In International Conference on Computer Vision, pages 1583-1590, 2011.

[18] F. Raimbault and Y. Incesu. Adaptive video stabilisation with dominant motion layer estimation for home video and TV broadcast. In IEEE International Conference on Image Processing, 2013.

[19] S. Rao, R. Tron, R. Vidal, and Y. Ma. Motion segmentation via robust subspace separation in the presence of outlying, incomplete, or corrupted trajectories. In IEEE Conference on Computer Vision and Pattern Recognition, pages 1-8, 2008.

[20] A. Richtsfeld, M. Zillich, and M. Vincze. Implementation of Gestalt principles for object segmentation. In International Conference on Pattern Recognition, pages 1330-1333, 2012.

[21] M. Rubinstein and C. Liu. Towards longer long-range motion trajectories. In British Machine Vision Conference, pages $1-11,2012$.

[22] P. Sand and S. Teller. Particle video: Long-range motion estimation using point trajectories. In IEEE Conference on Computer Vision and Pattern Recognition, pages 2195-2202, 2006.

[23] J. Shi and J. Malik. Normalized cuts and image segmentation. IEEE Transactions on Pattern Analysis and Machine Intelligence, 22(8):888-905, 2000.

[24] J. Shi and C. Tomasi. Good features to track. In IEEE Conference on Computer Vision and Pattern Recognition, pages 593-600, 1994.

[25] N. Sundaram, T. Brox, and K. Keutzer. Dense point trajectories by GPU-accelerated large displacement optical flow. In European Conference on Computer Vision, pages 438-451, 2010.

[26] R. Tron and R. Vidal. A benchmark for the comparison of 3-D motion segmentation algorithms. In IEEE Conference on Computer Vision and Pattern Recognition, pages 1-8, 2007.

[27] L. Zappella, X. Lladó, E. Provenzi, and J. Salvi. Enhanced local subspace affinity for feature-based motion segmentation. Pattern Recognition, 44(2):454-470, 2011. 


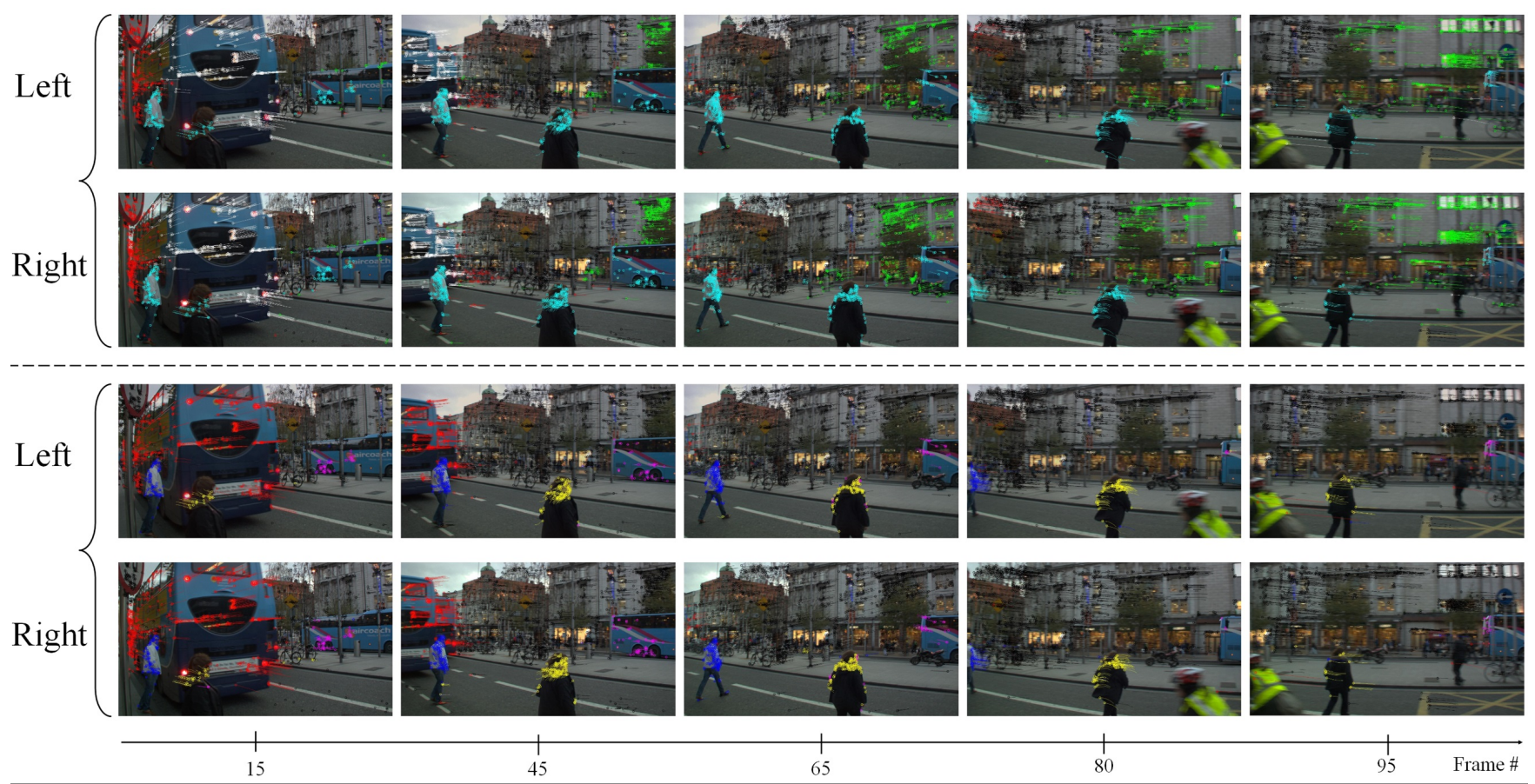

Figure 9: gpo_pan sequence - results of automatic divisive/agglomerative clustering with number of clusters set to 5 (top rows) and after user-assisted split-and-merge producing 5 clusters (14 split steps, 5 merge steps, 15 selections) (bottom rows).

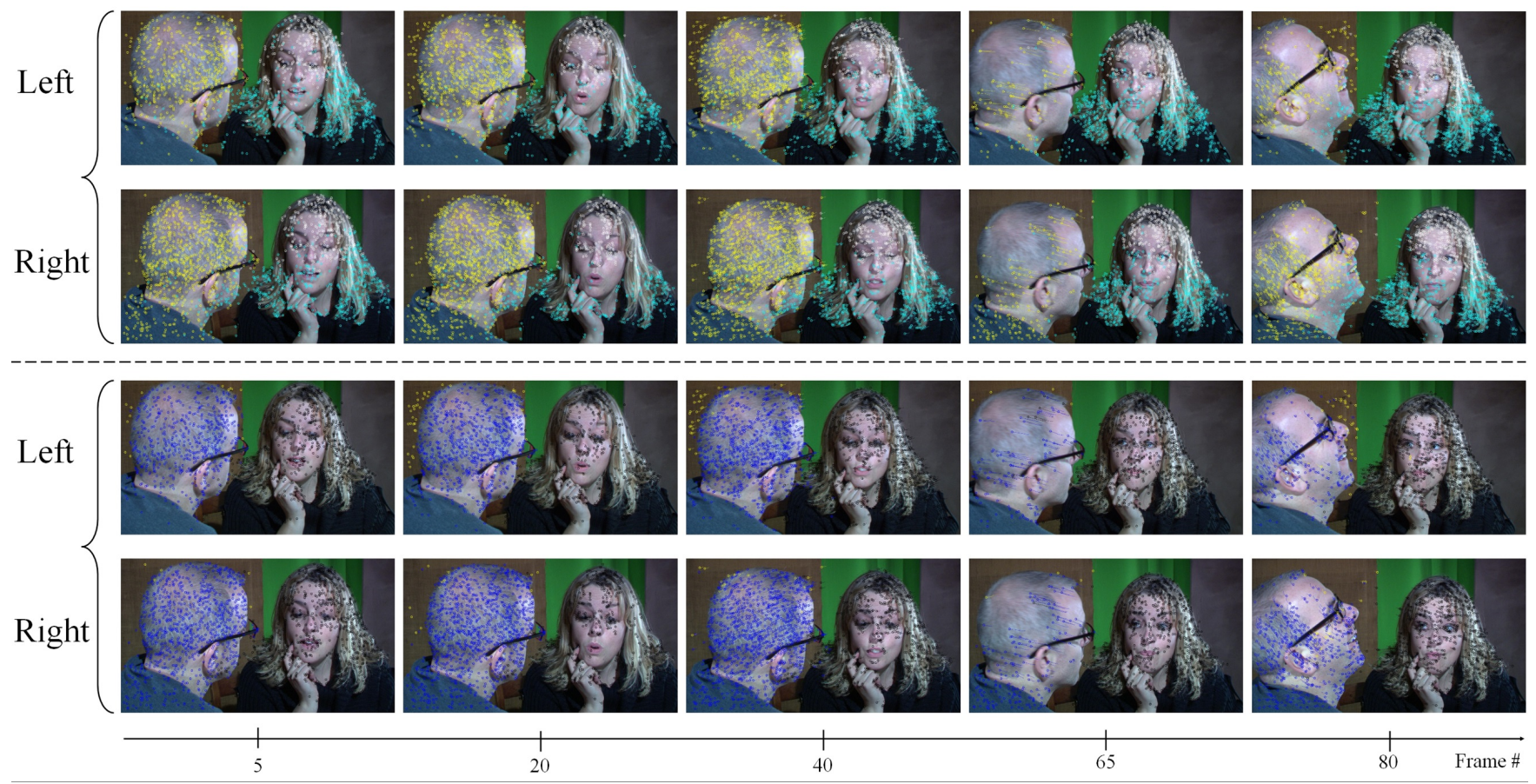

Figure 10: close_up_1 sequence - results of automatic divisive/agglomerative clustering with number of clusters set to 3 (top rows) and after user-assisted split-and-merge producing 3 clusters (17 split steps, 3 merge steps, 18 selections) (bottom rows). 